\title{
Analysis of the Microbiome and Mycobiome of Foot Skin in Patients with Diabetes Mellitus
}

\author{
Yang Won Lee, Joo Ran Hong, Hye In Cheon, Min Seok Hur, Song Hee Han, Byung Gon Choi, \\ Yong Beom Choe, Kyu Joong Ahn
}

\section{Department of Dermatology, Konkuk University School of Medicine, Seoul, Korea}

Background Recent studies have revealed that the composition of microbiome varies under distinct biological circumstances induced by altered host immunity or other pathologic state. Diabetes mellitus (DM) is one of the most prevalent endocrine diseases. We hypothesized that in diabetic patients, the impaired host immunity and changes in microenvironment caused by hyperglycemia and vascular impairment influence on the structures of skin microbiome.

Objectives We aimed to comparatively analyze the overall skin micro- and mycobiome between patients with type 2 diabetes and healthy individuals.

Methods We obtained skin swab samples from the foot (toes and interdigital space) of 17 patients with diabetes mellitus (DM) and 18 healthy controls (HC). Composition of bacteria and fungi at different taxonomic levels were analyzed using cultureindependent sequencing method followed by direct DNA extraction and PCR. The phylogenic markers used were V3 to V4 regions of $16 \mathrm{~S}$ rRNA gene for bacteria and internal transcribed spacer 2 regions of DNA for fungi.

Results The age and sex distributions were not significantly different between two groups. The results of alpha (within samples) and beta analyses (between samples) are shown in Figure 1 and 2, respectively.

Discussion We observed distinct characteristics of skin microand mycobiome between samples from diabetic patients and healthy controls. Further investigations and clinical correlations are needed. Imbalance in host-microbiota equilibrium and biofilm formation might be one of the contributing factors for chronic

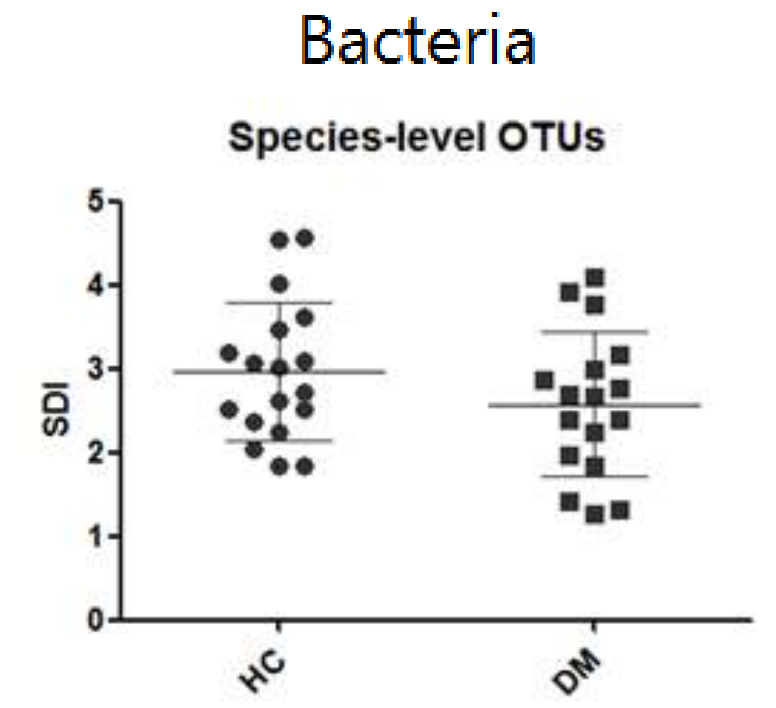

hylum in $>5 \%$ abundance

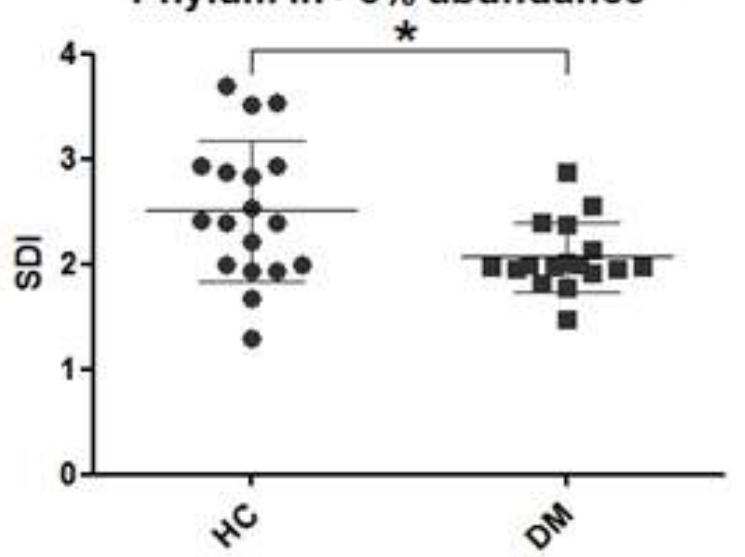

Species in $>5 \%$ abundance

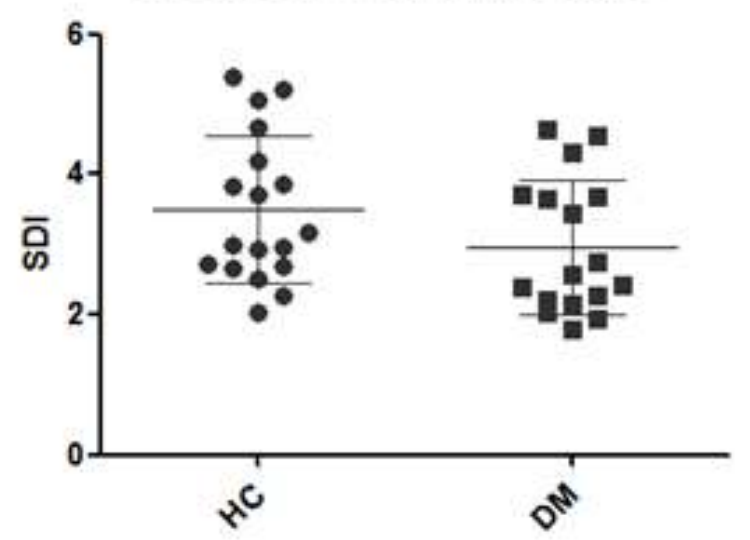

Figure 1 Diversity analyses using Shannon diversity index (SDI). SDls of each sample are marked as circular $(\mathrm{HC} 1-\mathrm{HC} 18)$ and square dots (DM1-DM17). Statistically, the data were analyzed using unpaired $t$-test and the standard bar represents the mean \pm SD. ${ }^{*} p<0.05$. OUT: operating taxonomic unit; $\mathrm{HC}$ : healthy control; DM: diabetes mellitus.

non-healing wounds in diabetic patients with peripheral neuropathy and vasculopathy.

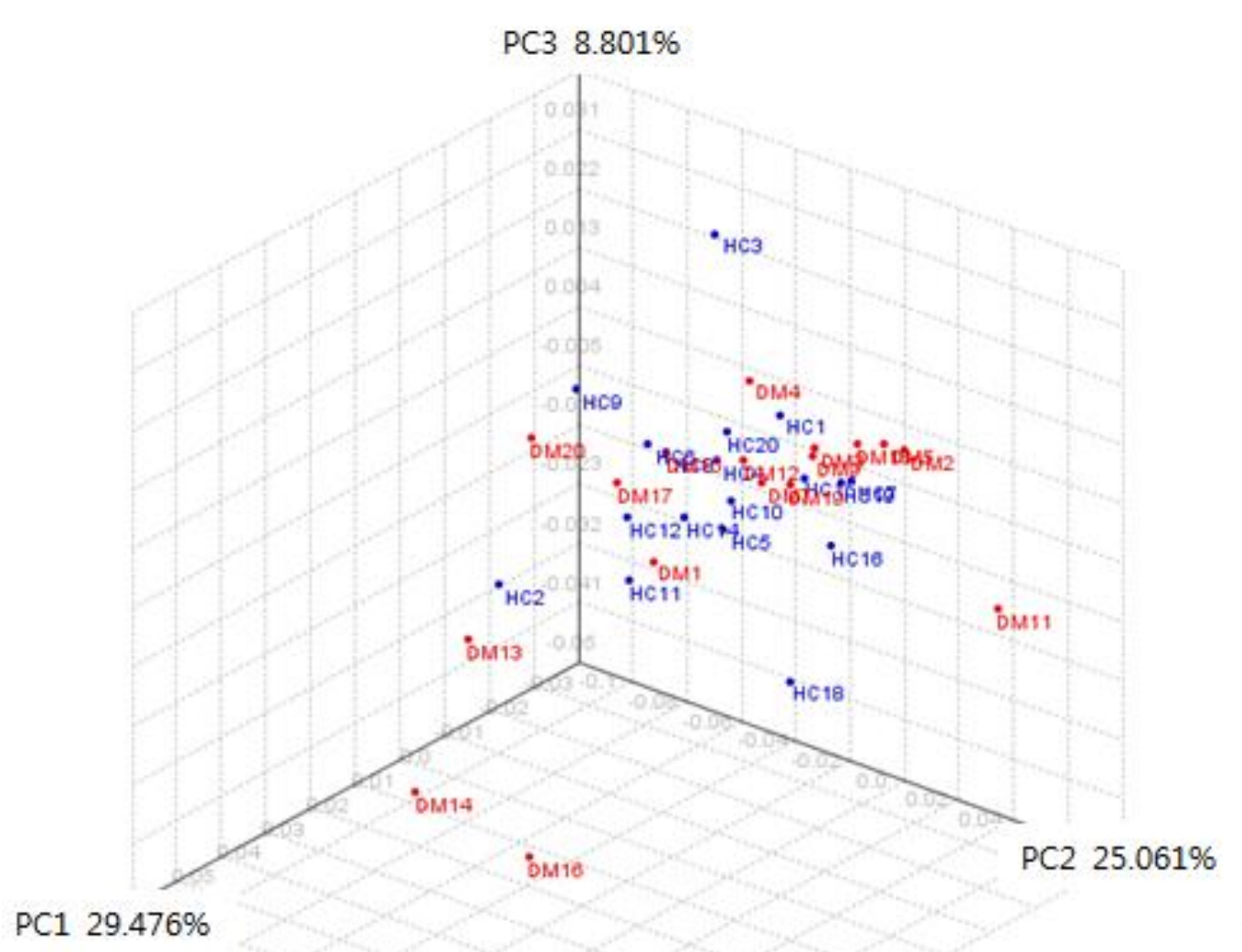

Bacteria
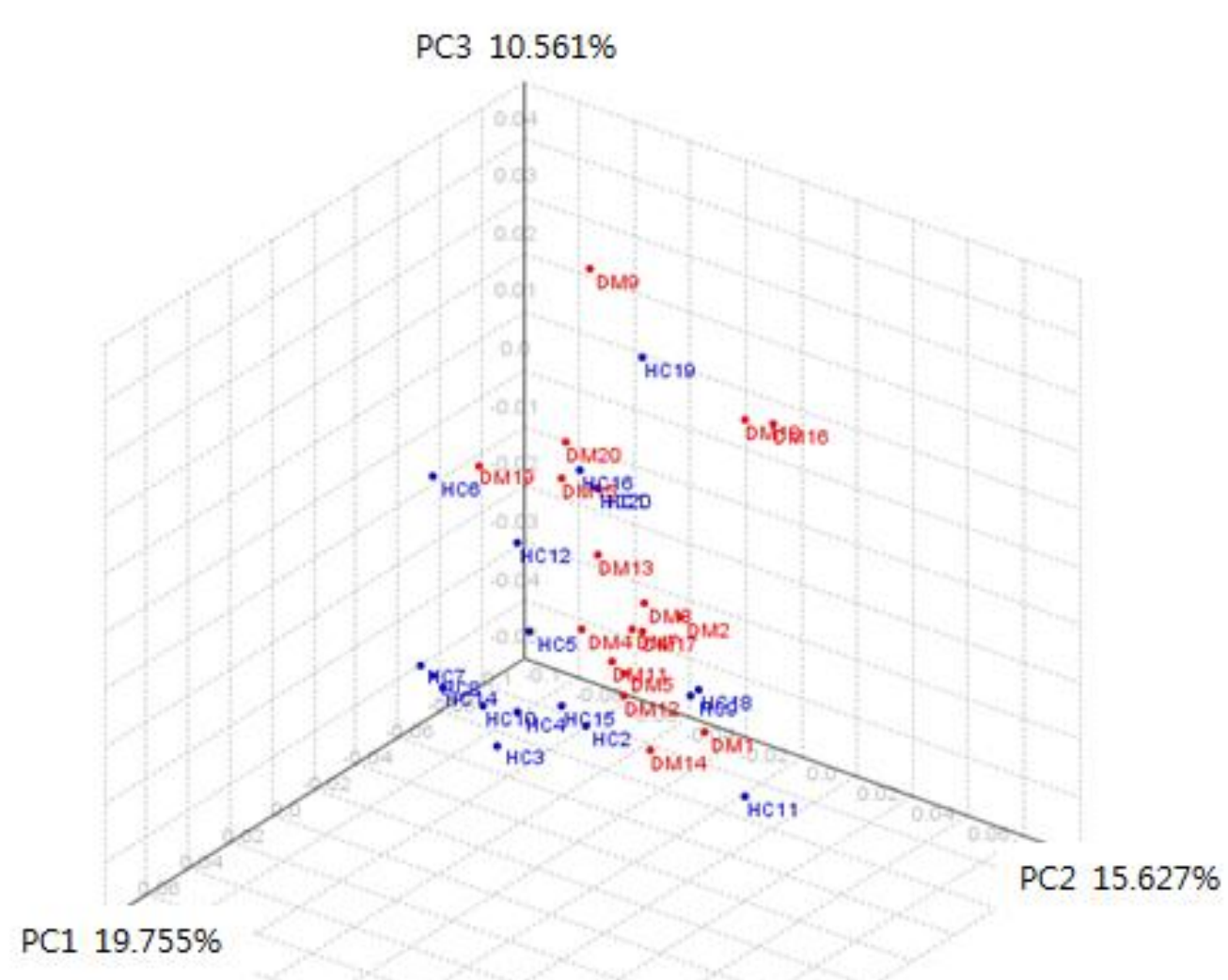

Fungus
Figure 2 Principal coordinate analysis (PCoA) plot using weighted Fast Unifrac analysis.

This plot represents beta-diversity comparisons of the micro- and mycobiomes of samples, based on a phylogenetic distance. Not only the presence or absence of OTUs but also the relative abundance of OTUs was considered. The red dots indicate DM samples (DM1-DM17), and the blue dots represent healthy controls $(\mathrm{HC} 1-\mathrm{HC} 18)$. Three major axes are shown with the respective percentages of explained variance. The DM samples were considerably more differentiated in bacterial communities compared to samples from healthy controls. The samples tended to show clustering of OTUs in accordance with the presence or absence of DM, though there was some overlap between the groups. OUT: operating taxonomic unit; $\mathrm{HC}$ : healthy control; DM: diabetes mellitus. 